\title{
O USO DA REALIDADE AUMENTADA COM DISPOSITIVOS MÓVEIS NA EDUCAÇÃO MATEMÁTICA COMO POTÊNCIA NA GEOMETRIA ESPACIAL
}

\section{LUIS OTONI MEIRELES RIBEIRO}

Instituto Federal de Educação, Ciência e Tecnologia Sul-Rio-Grandense (IFSUL). Pós-doutor em Educação na UFSC. Doutor em Informática na Educação na UFRGS. Mestre em Tecnologia - Educação Tecnológica no CEFET-PR/UTFPR. Professor Titular do Mestrado em Educação e Tecnologia - MPET. ORCID: 0000-0002-5526-8632 E-mail: luisribeiro@ifsul.edu.br

\section{LISANDRA XAVIER GUTERRES}

Instituto Federal de Educação, Ciência e Tecnologia Sul-Rio-Grandense (IFSUL). Bacharel em Design Gráfico na UFPEL. Mestranda em Educação e Tecnologia - MPET. ORCID: 0000-00019432-8864 E-mail: 1.xguterres@gmail.com

\section{DENISE NASCIMENTO SILVEIRA}

Instituto Federal de Educação, Ciência e Tecnologia Sul-Rio-Grandense (IFSUL). Pós-doutora na Universidade do Porto. Doutora em Educação. Mestre em Educação. Graduação no Instituto de Física e Matemática - IFM. Departamento de Matemática e Estatística - DME. Professora titular do Mestrado em Educação e Tecnologia - MPET. ORCID: 0000-0001-9951-2302.

E-mail: silveiradenise13@gmail.com 


\section{O USO DA REALIDADE AUMENTADA COM DISPOSITIVOS MÓVEIS NA EDUCAÇÃO MATEMÁTICA COMO POTÊNCIA NA GEOMETRIA ESPACIAL}

Este texto apresenta o uso da realidade aumentada em dispositivos móveis como um recurso educacional potencial para o ensino de Geometria Espacial, pois essa tecnologia disruptiva permite que o aluno se aproxime do objeto de aprendizagem, através da visualização interativa e imersiva de conceitos abstratos da matemática. Visto que a realidade aumentada possibilita a manipulação de objetos virtuais tridimensionais, os quais podem são visualizados sobrepostos ao ambiente físico, esse texto argumenta como a ferramenta pode ser utilizada no ensino de Geometria Espacial, com o objetivo de promover experiências significativas aos alunos pela manipulação de sólidos geométricos em seus dispositivos móveis..

Palavras-chave: Geometria Espacial. Realidade Aumentada. Tecnologias Educacionais.

\section{EL USO DE LA REALIDAD AUMENTADA CON DISPOSITIVOS MÓVILES LA EDUCACIÓN MATEMÁTICA COMO GEOMETRÍA ESPACIAL}

Este texto presenta el uso de la realidad aumentada en dispositivos móviles como un recurso educativo potencial para la enseñanza de la Geometría Espacial, ya que esta tecnología disruptiva permite al estudiante acercarse al objeto de aprendizaje, a través de la visualización interactiva e inmersiva de conceptos matemáticos abstractos. Dado que la realidad aumentada permite manipular objetos virtuales tridimensionales, que se pueden ver superpuestos en el entorno físico, este texto argumenta cómo se puede utilizar la herramienta en la enseñanza de la Geometría Espacial, con el fin de promover experiencias significativas para los estudiantes mediante la manipulación sólidos geométricos en sus dispositivos móviles.

Palabras clave: Geometría espacial. Realidad aumentada. Tecnologías educativas.

\section{THE USE OF AUGMENTED REALITY WITH MOBILE DEVICES IN MATHEMATICS EDUCATION AS A POWER IN SPATIAL GEOMETRYN}

This text presents the use of augmented reality on mobile devices as a potential educational resource for the teaching of Spatial Geometry, as this disruptive technology allows the student to get closer to the learning object, through the interactive and immersive visualization of abstract concepts in mathematics. Since augmented reality makes it possible to manipulate three-dimensional virtual objects, which can be viewed superimposed on the physical environment, this text argues how the tool can be used in the teaching of Spatial Geometry, with the aim of promoting meaningful experiences to students by manipulating geometric solids on your mobile devices.

Keywords: Spatial Geometry. Augmented Reality. Educational Technologies.

\section{plurais}




\section{O USO DA REALIDADE AUMENTADA COM DISPOSITIVOS MÓVEIS NA EDUCAÇÃO MATEMÁTICA COMO POTÊNCIA NA GEOMETRIA ESPACIAL}

\section{Introdução}

A evolução tecnológica possibilitou inúmeros avanços e facilidades às atividades realizadas pela população, como o acesso à tecnologia, provinda da inserção dispositivos móveis que, pelas suas características práticas e econômicas, tornaram-se recursos amplamente utilizados pelas pessoas, contribuindo na facilitação do desempenho de suas atividades cotidianas. No Brasil, conforme dados da Fundação Getúlio Vargas de São Paulo (ESTADO DE MINAS, 2019), em 2019 foi detectado que para cada habitante existem dois dispositivos digitais, incluindo smartphones, tablets e notebooks, dos quais se destacam o uso de smartphones, sendo 230 milhões de celulares ativos no país, em contrapartida o número de notebooks, tablets e computadores são de 180 milhões. Os dados revelam que os smartphones estão cada vez mais presentes e são o caminho mais próximo entre a população e o acesso à tecnologia e a Internet.

Com base nesses dados, percebe-se que a amplitude do acesso aos dispositivos móveis, permite que usuários estabeleçam conexão com os aplicativos criados para serem utilizados nesses equipamentos. Existem inúmeros aplicativos destinados a diversas funções, como fazer compras online, realizar videoconferências, controlar contas bancárias, entre outros usos, nos quais se destaca a educação.

Um dos aspectos positivos do uso de aplicativos móveis na educação é a possibilidade de inserir tecnologias como Realidade Aumentada e Realidade Virtual no contexto educacional. Essas tecnologias, até então, estavam pouco difusas em função da complexidade dos sistemas, os quais necessitavam de grandes aparatos tecnológicos para existir. Contudo, com a adequação para o uso em aplicativos mobile, foram quebradas essas barreiras de acesso, permitindo à população conhecer e utilizar a RV e RA em diferentes contextos.

Entre as finalidades de uso dos aplicativos de RA e RV, destaca-se a utilização na educação de matemática, pois essas tecnologias disruptivas permitem a inserção de conteúdos educacionais 
em um contexto digital, no qual o aluno pode visualizar, de forma imersiva e interativa, conceitos abstratos, permitindo uma aproximação do aluno com o objeto de aprendizagem.

A Realidade Aumentada se caracteriza, conforme Kirner e Kirner (2008) pela inserção de objetos virtuais nos ambientes físicos que são apresentados aos usuários, em tempo real, com o apoio de um dispositivo tecnológico (Figura 1) que utiliza como interface o ambiente real. Assim, a inserção dos objetos virtuais no espaço físico pode ocorrer pela captura, com uso da câmera do dispositivo, da imagem de um marcador que é processada na aplicação instalada e, posteriormente, exibe um modelo tridimensional, vídeo ou imagem previamente associada ao marcador na tela do dispositivo ou a partir da localização geográfica do usuário, liberando informações conforme os dados de localização.

Figura 1 - Aplicativo de Realidade Aumentada

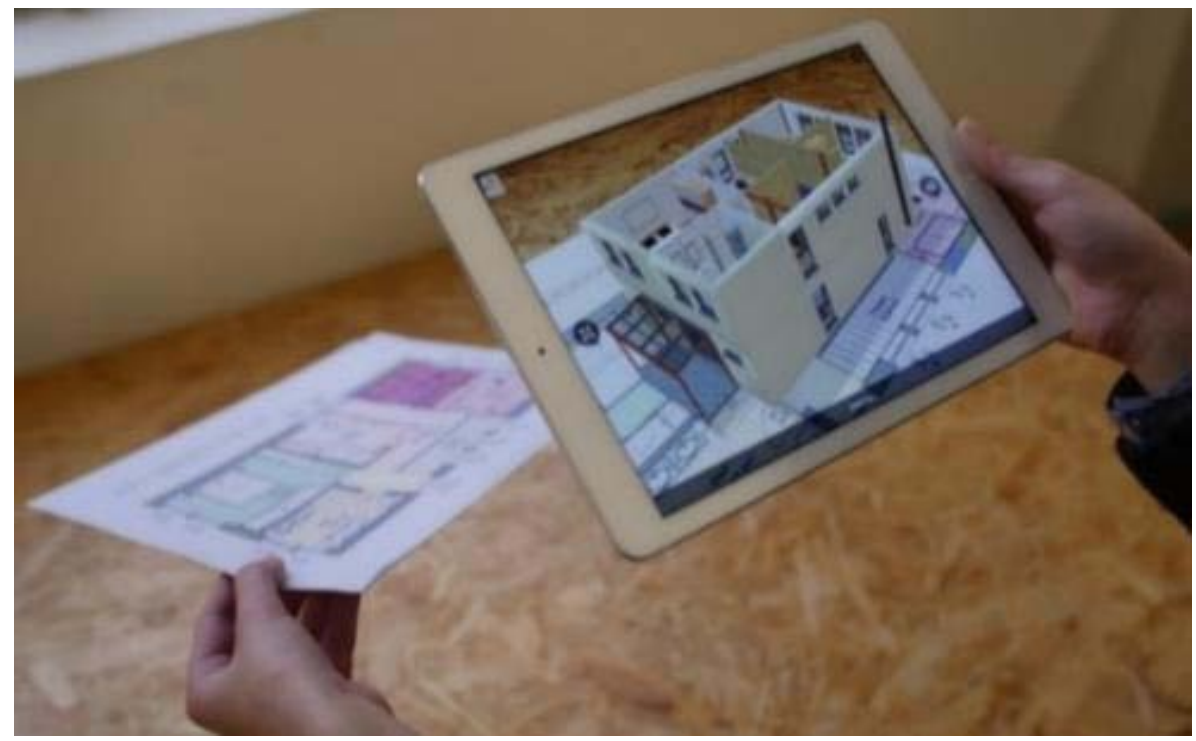

Fonte: Blog da Arquitetura (2020) ${ }^{1}$

1 Blog da Arquitetura. Disponível em: <https://www.blogdaarquitetura.com/augment-o-app-que-une-3d-e-realidade-aumentada/>. Acesso em: 31 mai. 2020.

\section{plurais}


Já a Realidade Virtual permite a imersão de um usuário, em tempo real, em um ambiente virtual tridimensional, ou seja, diferentemente da RA que potencializa o ambiente real, a RV suprime o espaço físico, utilizando apenas o ambiente sintético.

Com base nos aspectos dessas tecnologias este estudo tem como objetivo abordar o uso da Realidade Aumentada no ensino de Geometria Espacial. Para isso, foram apontadas algumas das principais possibilidades que podem ser contribuições significativas do uso da RA no ensino de sólidos geométricos.

Conforme Marques et al. (2018), existem diversos fatores que podem implicar nas dificuldades no ensino em matemática, como as metodologias adotadas pelos docentes, muitas vezes consideradas desinteressantes ou até mesmo ultrapassadas pelos alunos. Por isso, torna-se imprescindível rever as estratégias de ensino e aprendizagem utilizadas com os discentes, de forma a incluir atividades que engajem o estudante e facilitem sua compreensão.

A partir das dificuldades percebidas no ensino de Geometria Espacial, foram abordadas teoricamente nesta pesquisa as possíveis contribuições da Realidade Aumentada no ensino de matemática.

\title{
O Ensino de Geometria Espacial
}

Segundo Eves (2004) a matemática primitiva surgiu no Oriente Antigo como uma ciência prática para auxiliar as atividades ligadas à agricultura e à engenharia, pois essas atividades necessitavam da realização de cálculos para calendários utilizáveis, desenvolvimento de sistemas de pesos e medidas nas colheitas, criação de métodos de agrimensura para a construção de canais e reservatórios, entre outros aspectos. Com base nisso, desenvolveram-se tendências no sentido da abstração e, de certa forma, iniciou-se o estudo da ciência em si.

Além das práticas relacionadas ao plantio e engenharia, acredita-se que o estudo da geometria ocorreu no século XX a.C., nas civilizações egípcia e babilônica, em função da construção monumentos como as pirâmides e outros, os quais não seriam possíveis de serem realizado sem os devidos conhecimentos geométricos (EVES, 2004).

\author{
plurais


Desde os primórdios da antiguidade a geometria está extremamente vinculada às atividades cotidianas da população. Sendo assim, torna-se impossível dissociá-la da realidade, pois esses conceitos abstratos, na verdade, fazem parte de tudo ao nosso redor, contudo, as práticas educacionais no ensino de matemática que se utilizam apenas de conceitos abstratos, como uso de cálculos e formas, acabam dificultando o entendimento, dos estudantes fazendo com que os alunos tenham dificuldades no aprendizado.

De acordo com o Ministério da Educação (MEC) (BRASIL, 1998, p.19), “o ensino de Matemática ainda é marcado pelos altos índices de retenção, pela formalização precoce de conceitos, pela excessiva preocupação com o treino de habilidades e mecanização de processos sem compreensão.”. Esse aspecto da abordagem adotada no ensino, pode ser uma das razões do distanciamento na relação entre matemática e realidade.

Por isso, se faz necessário o uso de discussões no âmbito da Educação Matemática que adequem o sistema escolar de ensino a uma realidade na qual os conceitos desta disciplina devam estar vinculados a diversos campos da atividade humana. E, nessa perspectiva, o campo da Educação Matemática vem se desenvolvendo como uma forte área do conhecimento que busca essas aproximações, principalmente com o uso das tecnologias.

Desta forma, é perceptível que a realidade aumentada é uma estratégia que pode propiciar aos alunos o estabelecimento de relações, através de meios digitais pela aproximação da matemática com elementos do cotidiano, explorando todos os seus aspectos práticos, pois a RA permite que o usuário manipule objetos virtuais tridimensionais e os introduzam em ambientes reais, gerando experiências significativas aos alunos.

Outro aspecto para se considerar é que existem algumas dificuldades no ensino de geometria que podem decorrer de fatores associados às fases do desenvolvimento da criança. Piaget e Inhelder (1981, p. 167) realizaram um estudo no qual são analisados os mecanismos da construção do espaço na criança. Conforme os autores as primeiras relações que a criança desenvolve estão relacionadas as de ordem topológica, em que são definidas as noções de vizinhança, separação, ordem, envolvimento, continuidade e grandezas. Contudo, ao contrário do espaço topológico, o espaço projetivo e o espaço euclidiano consistem em situar os objetos com relação a outros, 
conforme suas projeções, perspectivas ou em "coordenadas". Pelo fato de serem mais complexas, essas estruturas são construídas mais tardiamente pela criança.

O espaço projetivo e o espaço euclidiano apoiam-se um no outro, pois a construção dos sistemas naturais de coordenadas, que marcam o acabamento das noções euclidianas fundamentais, nos parece sincronizar com a coordenação geral dos pontos de vista característicos do espaço projetivo. (PIAGET; INHELDER, 1981, p.438).

Com base na reflexão dos aspectos estabelecidos pelos autores, percebe-se que existem períodos propícios para o desenvolvimento de certas habilidades matemáticas na criança, que de forma geral, ocorrem com pequenas variações de idades. Contudo, nesses períodos podem ocorrer bloqueios e dificuldades na compreensão e estimulação do desenvolvimento dessas habilidades, resultando em experiências negativas que afetam a compreensão dessa ciência. Sendo assim, é importante propiciar práticas em diferentes níveis que permitam o desenvolvimento da criança de acordo com a fase que ela está vivenciando, mas sempre que possível relacionando com elementos do cotidiano para que o indivíduo possa construir estruturas cognitivas com mais facilidade para o novo conhecimento.

Os recursos digitais estão cada vez mais presentes nas famílias, inclusive com as crianças, que utilizam dispositivos móveis para assistir desenhos, jogar, comunicar-se, entre outros aspectos. Os smartphones, por exemplo, são um dos principais mecanismos para acessar o mundo digital. Então, permitir que a criança aprenda através do uso de um dispositivo que lhe é familiar pode ser uma estratégia eficaz. Contudo, a tecnologia por si só não é suficiente, é necessário utilizar esse recurso com uma intencionalidade pedagógica.

É nesse ponto que a realidade aumentada e o ensino de geometria podem se aliar para estabelecer uma experiência de aprendizagem mais significativa ao alunos, pois permitir que os alunos manipulem objetos virtuais em conjunto com o ambiente real "pode ser concebido como uma transformação do campo perceptivo e todo campo perceptivo como um conjunto de relações determinadas por movimentos." (PIAGET; INHELDER, 1981, p.29).

O movimento com o objeto virtual, mesmo que não seja tátil, propriamente dito, existe enquanto manipulação do dispositivo móvel e do marcador do objeto virtual, manifestando uma resposta imediata às ações do usuário que podem ser visualizadas no display do dispositivo. Per-

\section{plurais}


ceber como as formas se comportam é um elemento fundamental para a aprendizagem de geometria espacial, afinal, ver o porquê e como ocorrem os cálculos com base nas experiências reais e conhecidas (subsunçores) podem tornar a aprendizagem mais significativa (AUSUBEL, 2003).

Dessa forma, infere-se que muitas das dificuldades na compreensão da geometria espacial são consequências da abordagem de forma inadequada de seu ensino. E, a realidade aumentada mostra-se como um caminho promissor para o processo de ensino e aprendizagem. Piaget e Garcia (2011) escrevem que quando as relações são compreendidas, elas obedecem às leis de compensações, pois as partes do objeto que se tornam invisíveis, em caso de rotação, são substituídas por partes até então invisíveis que se tornam visíveis; condições que pode ser oferecida pela realidade aumentada.

\section{A Realidade Aumentada}

O Termo "Realidade Aumentada" surgiu em 1990, quando o Prof. Thomas Caudell designou como Realidade Aumentada um projeto que estava desenvolvendo de um mostrador digital para aviões, que mesclava gráficos virtuais em uma realidade física, em colaboração com a empresa Boing.

Segundo Azuma (1997) a RA é uma tecnologia que combina os conteúdos reais e virtuais, que interagem concomitantemente e em tempo real, acrescentando elementos a realidade, em vez de substituí-la completamente. Em seu artigo "A Survey of Augmented Reality" o autor destaca três características fundamentais para classificar um sistema de RA:

a) Combinar conteúdo virtual e real;

b) O sistema deve ser interativo e a interação deve ocorrer em tempo real;

c) Alinhar elementos reais e virtuais em três dimensões.

No contexto atual, existem duas formas mais usuais de acionar os sistemas de realidade aumentada em aplicativos para dispositivos móveis, a primeira através do uso de marcadores, imagens bidimensionais que quando reconhecidas pelo uso da câmera do dispositivo em conjunto com a aplicação instalada nesse, exibem na tela modelos em realidade aumentada. A segunda se 
caracteriza pelo uso do posicionamento GPS para mapear a posição espacial do usuário e, assim, disponibilizar conteúdos dirigidos para aquela localização.

A crescente popularização da realidade aumentada pode ser atribuída ao surgimento do jogo Pokémon Go (Figura 2), desenvolvido em 2016 pela Niantic, que propiciou uma rápida disseminação mundial da RA. O Pokémon Go mostrou que é possível unir o real e o virtual, pois sua jogabilidade consiste em estimular as pessoas a saírem de casa para buscar novos desafios. Os usuários devem se dirigirem a pontos estratégicos para realizar a coleta de itens, tais como: pontos turísticos, museus, praças, monumentos, entre outros, incentivando a valorização da cultura de cada região.

Figura 2 - Pokémon Go
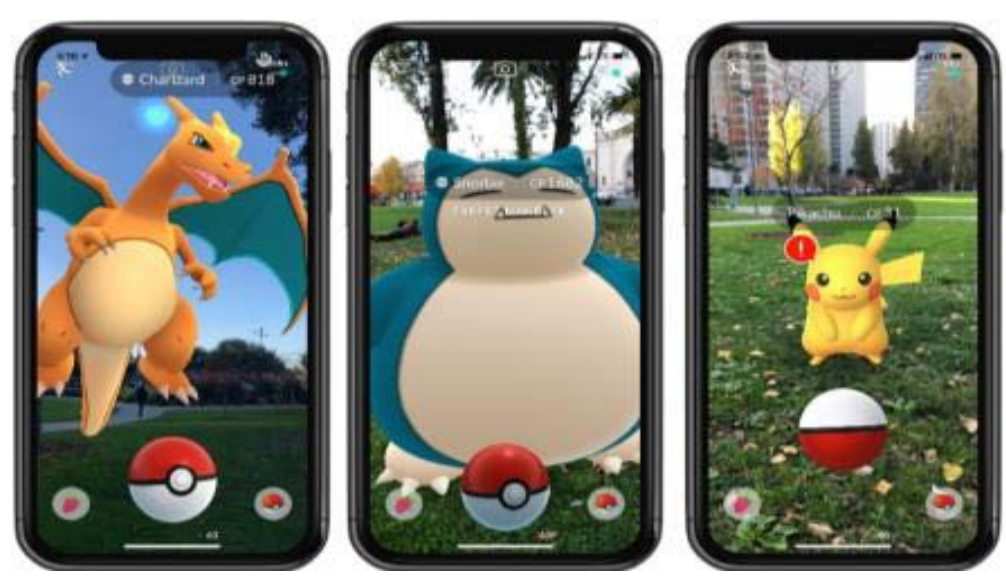

Fonte: Pokémon Go Live $(2020)^{2}$

Atualmente, existem softwares que permitem o desenvolvimento de aplicações de realidade aumentada e virtual de forma gratuita, como o Blender e Unity, ou com versões gratuitas para estudantes, como o 3DS Max Studio.

O Blender e o 3DS Max são programas de modelagem tridimensional que permitem modelar, mapear, texturizar, animar, iluminar e renderizar maquetes virtuais. Esses softwares trabalham em consonância com o Unity, um motor de jogo, desenvolvido pela Unity Technologies em 2005, que permite a importação de modelos tridimensionais de softwares como 3Ds Max, Blender, entre

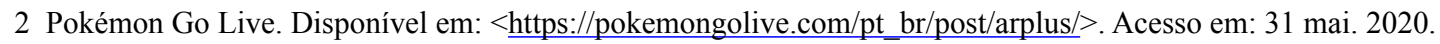

\section{plurais}


outros. Os modelos tridimensionais podem serem transformados em aplicações de RA ou RV para dispositivos móveis com o uso da Unity em conjunto com kits de desenvolvimento.

Existem diversas formas de desenvolver aplicações de RV e RA no Unity, por exemplo, a empresa Google disponibiliza o código aberto para criar experiências imersivas de realidade virtual para plataformas Android e iOS, através do uso do Google Cardboard (Figura 3) óculos de realidade virtual feito de papelão para realidade virtual que também tem seu molde disponibilidade pela Google. A Google também oferece o ARCore, um kit de desenvolvimento gratuito para gerar aplicações de realidade aumentada. Contudo, as aplicações desenvolvidas com uso do ARCore podem ser utilizadas apenas em dispositivos móveis mais atuais com Android 7.0 ou superior.

Figura 3 - Google Cardboard

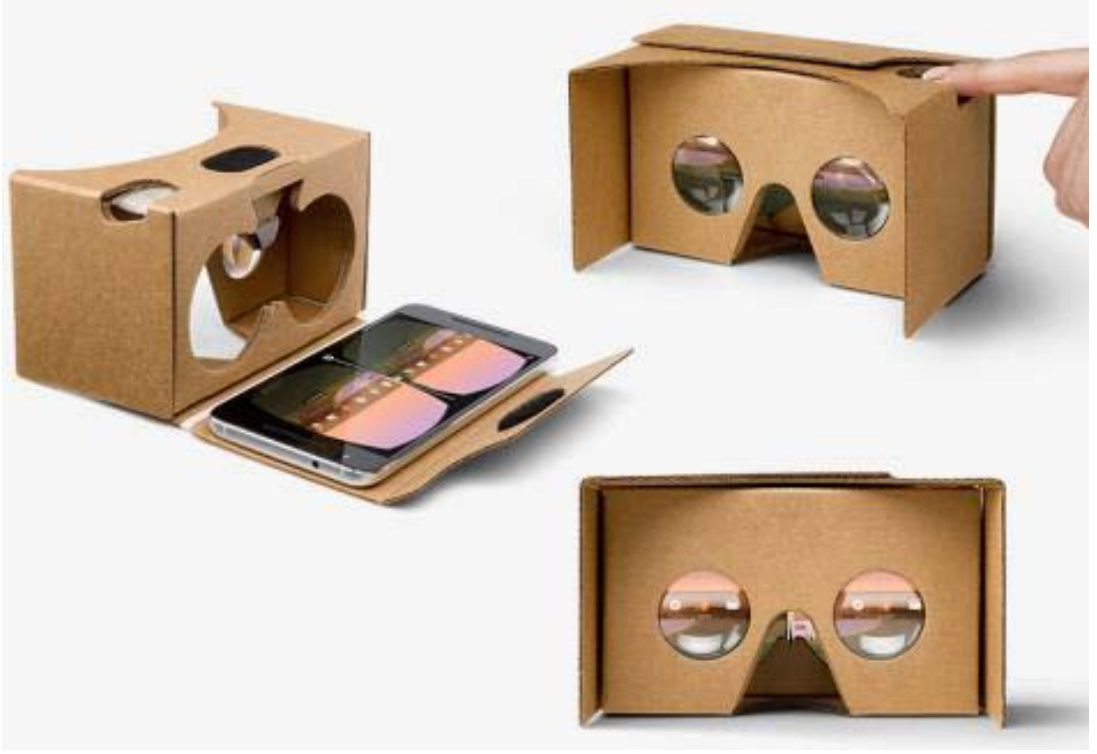

Fonte: ARVR Google $(2020)^{3}$

O Vuforia é um dos mais populares kits de desenvolvimento para aplicações de realidade aumentada. Entretanto, possui apenas uma versão gratuita para desenvolvedor, ou seja, não é possível a distribuição dos aplicativos gerados a partir dele, para isso é necessário adquirir uma chave

3 ARVR Google. Disponível em: <https://arvr.google.com/cardboard/>. Acesso em: 28 jun. 2020.

\section{plurais}


de licença paga. Contudo, devido a sua facilidade de uso e por permitir o desenvolvimento de uma aplicação de RA para dispositivos com Android inferior ao 7.0, que quando instaladas diretamente no dispositivo, ou seja, não distribuídas em lojas virtuais ou comercialmente, podem ser utilizadas gratuitamente. Tal facilidade permite, assim, que professores e alunos obtenham uma experiência de geração de aplicativos de RA de maneira fácil e acessível.

\section{Realidade Aumentada e Ensino de Geometria Espacial}

Além da possibilidade dos próprios educadores e alunos desenvolverem softwares de RA, como os recursos vistos no capítulo anterior, existem aplicativos criados e distribuídos gratuitamente para o ensino de geometria espacial que utilizam realidade aumentada. Dentre eles, destacamos:

\section{GeometriAR}

O GeometriAR (Figura 4) é um aplicativo para o ensino de geometria espacial no qual é possível visualizar sólidos, como prismas, pirâmides, cilindros, cones, entre outros, em realidade aumentada a partir da captura de imagens pela câmera do dispositivo.

Figura 4 - GeometriAR

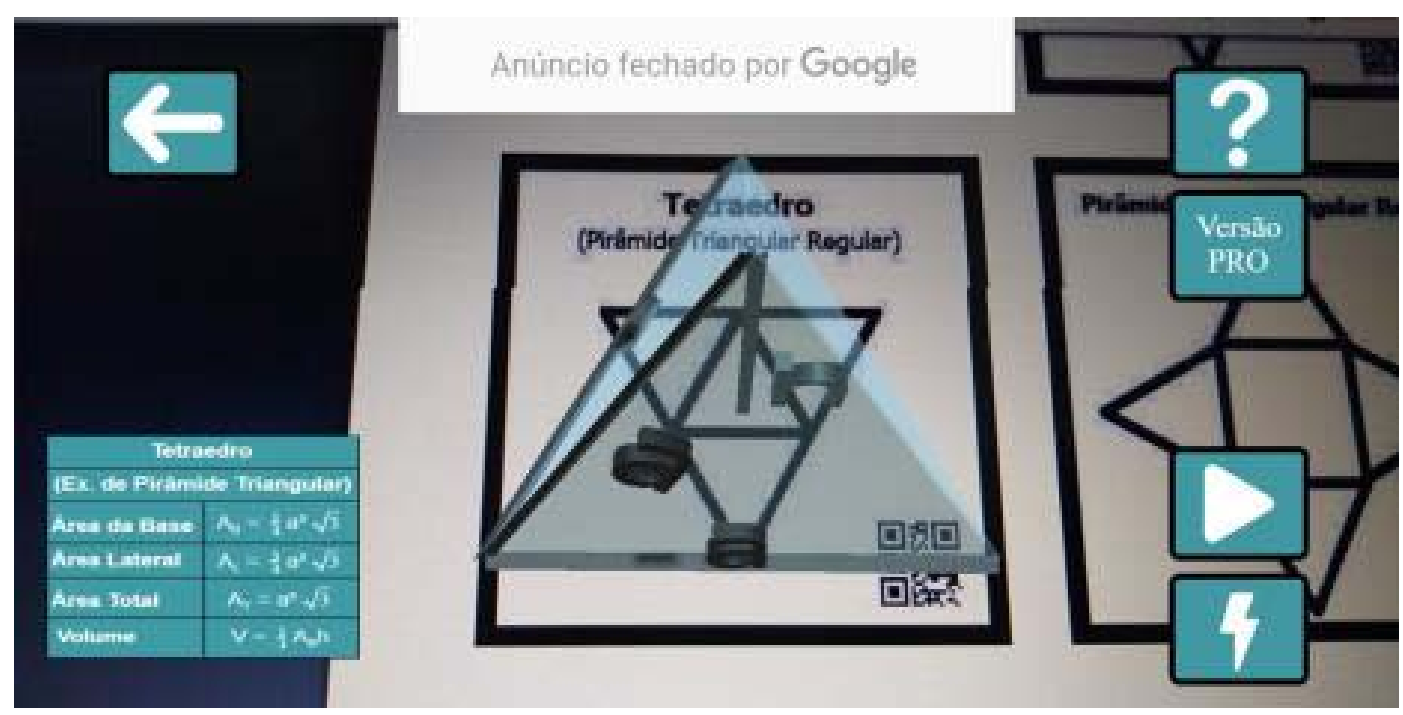

Fonte: Elaborado pelos autores com base na captura da tela do aplicativo GeometriAR

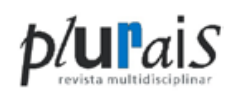

Salvador, v. 5, n. 2, p. 40-57, mai./ago. 2020 
Além disso, o aplicativo exibe animações com simulações da planificação dos objetos (Figura 5) e, também, fórmulas matemáticas relacionadas a cada sólido.

Figura 5 - Planificação de um cubo no GeometriAR

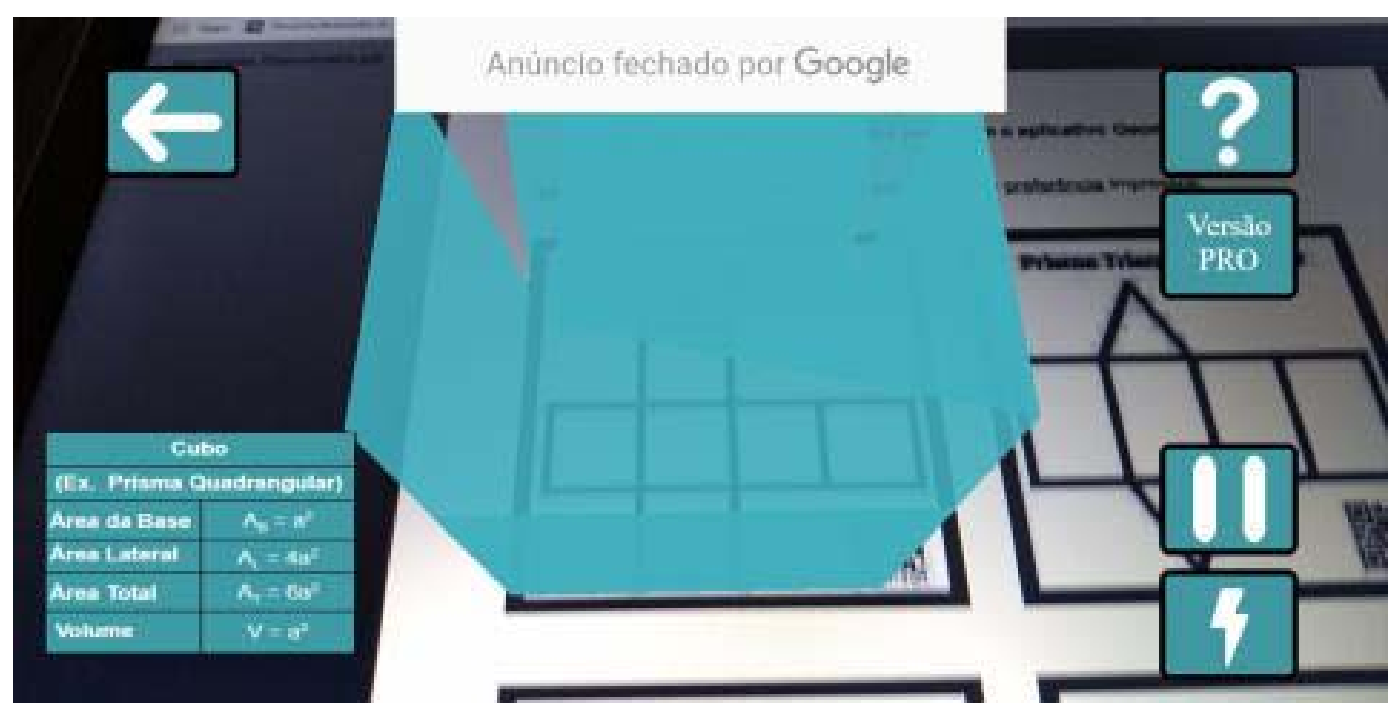

Fonte: Elaborado pelos autores com base na captura da tela do aplicativo GeometriAR

O aplicativo pode ser um importante aliado no ensino de geometria espacial, pois possibilita aos alunos explorar tridimensionalmente sólidos, além de permitir a planificação e o acesso às fórmulas correspondentes. Por fim, o GeometriAR propõe um desafio, com uma série de questões referentes aos sólidos geométricos. O aplicativo possui duas versões, uma gratuita e uma Pro. A versão Pro se distingue da gratuita por não apresentar anúncios e disponibilizar mais sólidos para análise.

\section{Polyèdres augmentès}

Assim como o GeometriAR, o Polyèdres augmentès (Figura 6) é um aplicativo que permite visualizar sólidos geométricos tridimensionais através da captura da imagem pela câmera dos dispositivos. 
Figura 6 - Visualização de sólidos geométricos no Polyèdres augmentès

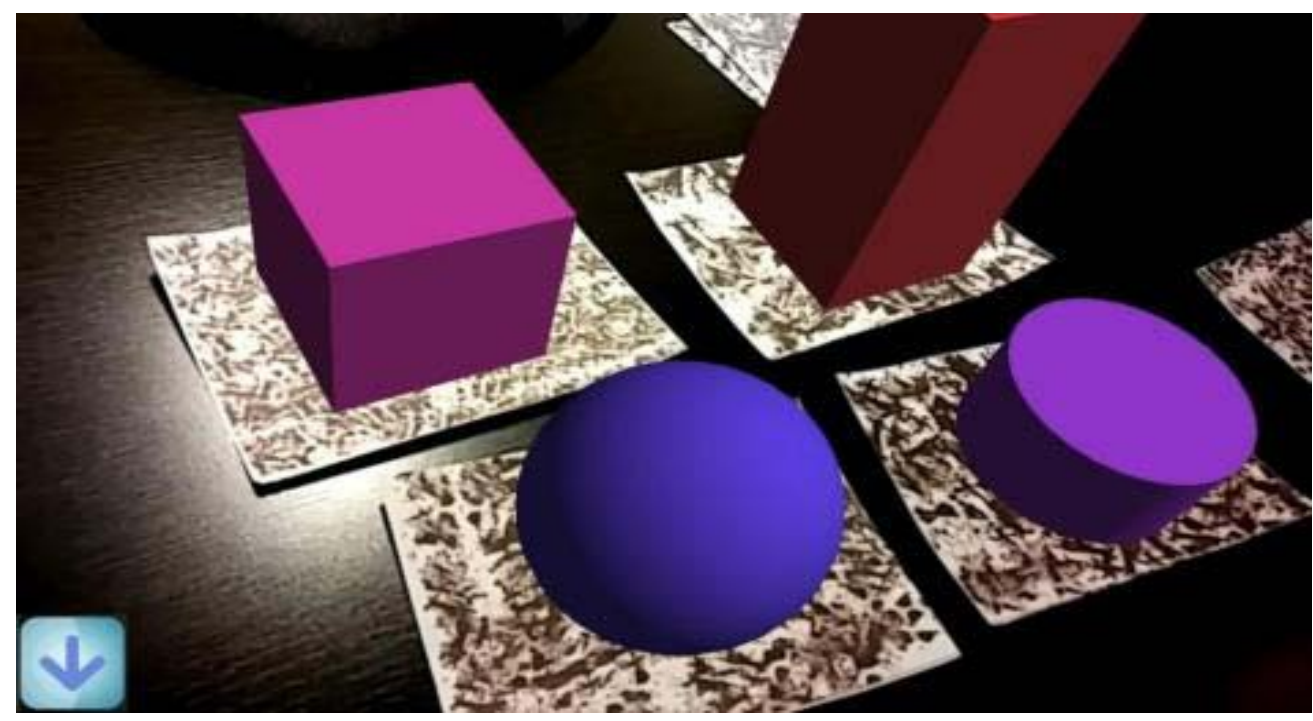

Fonte: Polyèdres augmentès $(2020)^{4}$

Nesse aplicativo é possível visualizar mais de um objeto simultaneamente na tela, por meio da captura da imagem de dois marcadores ou mais.

\section{Geometrix}

O Geometrix (Figura 7) é um aplicativo para visualização de sólidos geométricos que assim como os exemplos acima utiliza realidade aumentada e marcadores para disponibilizar o conteúdo. Além de permitir a visualização de modelos tridimensionais de poliedros regulares, pirâmides, prismas e sólidos de revolução em realidade aumentada, o aplicativo fornece informações sobre esses sólidos, tais como: número de faces, vértices, arestas, fórmula para calcular o volume e área externa.

4 Polyèdres augmentès. Disponível em: <https://play.google.com/store/apps/details?id=com.miragestudio. polygons\&hl=pt_BR $>$. Acesso em: 31 mai. 2020.

\section{plurais}


Figura 7 - Visualização de sólidos geométricos no Geometrix
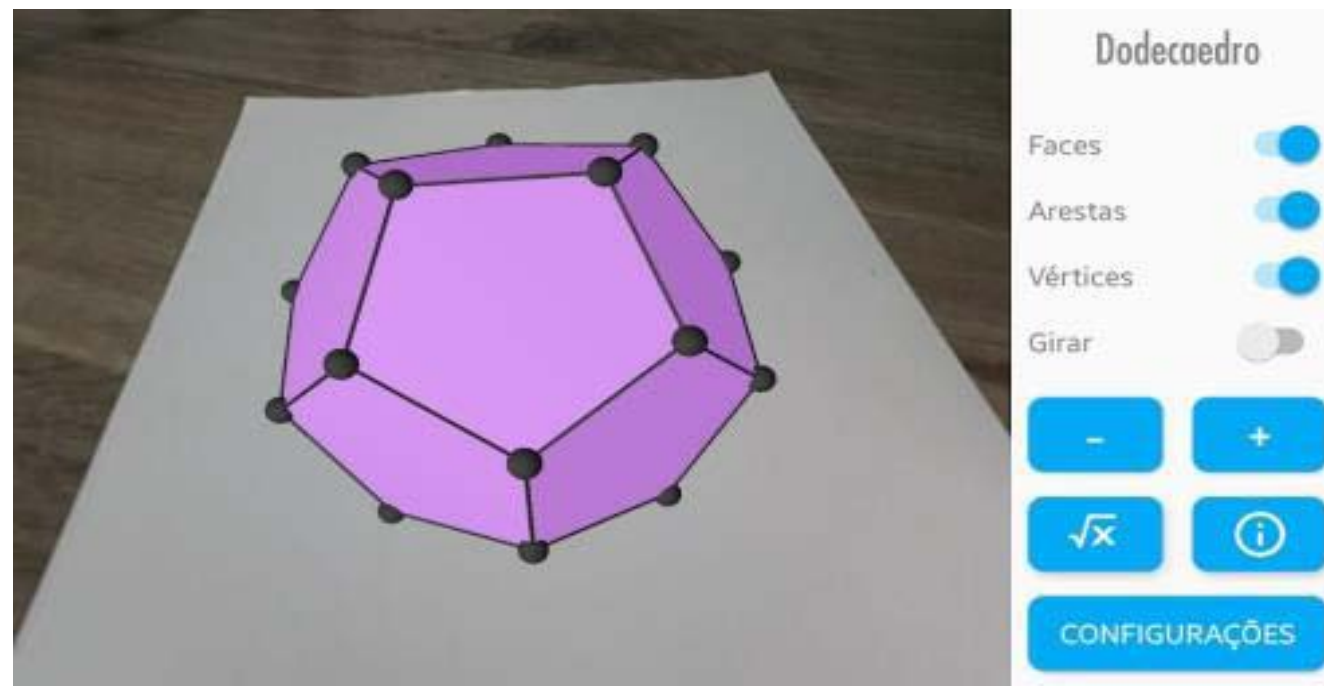

Fonte: Geometrix $(2020)^{5}$

As informações de faces, arestas e vértices podem ser acionadas ou omitidas, conforme as necessidades do usuário no menu direito do aplicativo. O usuário também pode habilitar ou desabilitar a opção de girar o sólido automaticamente, assim como aumentar ou diminuir o tamanho da figura, nos botões com o símbolo de mais e menos. No botão com a letra "i” são disponibilizadas mais informações, como a descrição da figura geométrica. No botão com o símbolo da raiz quadrada o usuário acessa as fórmulas, como volume e área do sólido em questão. Por fim, na opção configurações é possível selecionar a câmera frontal ou traseira, bem como a sua resolução.

Com base nos aplicativos apresentados acima foi possível perceber que existem uma série de funcionalidades disponibilizadas para auxiliar os alunos a compreender a geometria espacial através do uso de tecnologias móveis e realidade aumentada. Contudo, só a tecnologia não é o suficiente, pois sempre é necessário que o professor utilize os recursos com uma intencionalidade pedagógica específica, ou seja, propor práticas que possam unir a tecnologia aos conteúdos e atividades em sala de aula.

5 Geometrix. Disponível em: <https://play.google.com/store/apps/details?id=com.tcc.geometrix >. Acesso em: 31 mai. 2020.

\section{plurais}


A realidade aumentada permite ver os objetos virtuais simultaneamente a ambientes reais, possibilitando que o professor possa construir com os alunos a reflexão sobre soluções a problemas cotidianos, como a inclinação de uma rampa de acesso a entrada de uma escola, entre outros aspectos.

Conforme Ausubel (2003) o processo de aprendizagem significativa ocorre quando uma nova informação, apresentada de forma simbólica, estabelece um processo de ancoragem com os conhecimentos prévios do aprendiz (subsunçores), surgindo, com isso, um novo significado. Com base nisso, percebe-se que a Geometria Espacial pode utilizar da RA para exemplificar ações através do uso de recursos familiares aos indivíduos, como embalagens, monumentos, elementos do cotidiano, como a rampa de acesso descrita acima, além de outros objetos que podem ser construídos tridimensionalmente e transportados para a sala de aula, auxiliando no ensino de geometria espacial, através do uso da RA.

Também é importante ressaltar que pelo fato de ainda existir barreiras de acesso à tecnologia nas escolas, com a disseminação dos dispositivos móveis, os alunos podem utilizar os seus aparelhos para realizar as atividades. As barreiras podem ser devido à escassez e inacessibilidade aos laboratórios de informática que, por vezes, estão desativados em função de falta de manutenção, impossibilitando, assim, os alunos de experimentar recursos tecnológicos para a aprendizagem.

A adoção dessa prática se originou com a expressão Bring your own device (BYOD) ou traga o seu próprio dispositivo (tradução nossa) utilizado inicialmente pelos funcionários da empresa Intel (2009), que se refere ao ato de usar o seu próprio dispositivo móvel para acessar as informações. No contexto acadêmico, permitir que os alunos utilizem os seus dispositivos é uma estratégia que além de viabilizar o uso da tecnologia em sala de aula, promove a adoção de uma postura mais ativa dos estudantes, que visualizam e compartilham as suas experiências entre si, agindo sobre os objetos virtuais, o que permite uma experiência de "tangibilidade" (DE ALMEIDA; LIMA; BORGES, 2019).

Considerando que a matemática é uma ciência que possui muitos conceitos abstratos, essa característica tangível da realidade aumentada pode ser uma excelente estratégia para auxiliar na compreensão do conteúdo pelos alunos, pois conforme Piaget e Garcia (2011) o conhecimento não precede nem do sujeito consciente de si mesmo, nem dos objetos os quais se apropria. Essa ação

\author{
plurais


de construção do conhecimento é resultante das interações do sujeito com o meio, na proporção em que ele se apropria dos mecanismos internos de suas ações (BECKER, 1998).

Ainda, o autor ressalta a importância que a relação de movimento exerce, desde as fases primárias até o amadurecimento das relações entre objeto e espaço, "todo o movimento pode ser concebido como uma transformação do campo perceptivo e todo campo perceptivo como um conjunto de relações determinadas por movimentos." (PIAGET; INHELDER, 1981, p.29). Sendo assim, vemos uma contribuição significativa na utilização de realidade aumentada no ensino de matemática, pois a tecnologia permite estabelecer uma relação de movimento com o objeto virtual, mesmo que não seja tátil, propriamente dita, mas existente enquanto manipulação do modelo virtual em conjunto com o dispositivo móvel e o marcador, gerando respostas em tempo real as ações de rotação, aproximação e afastamento (escala) ou posição sobre o objeto virtual realizadas pelo usuário.

Por fim, permitir que os alunos experimentem essas tecnologias na educação possibilita que eles desenvolvam habilidades tecnológicas que são cada vez mais exigidas no contexto profissional, sendo assim, mediar o processo entre a educação e o uso da tecnologia é uma forma de preparar os estudantes para os desafios futuros. Tendo em vista o potencial das tecnologias de RA e RV, em que ambas possibilitam aos próprios estudantes ou professores desenvolverem modelos e compartilhá-los com a comunidade acadêmica, percebe-se que a RA e RV podem ser importantes recursos para a integração dos alunos com o meio digital.

\section{Considerações finais}

A revisão dos diversos aplicativos de Realidade Aumentada aqui exemplificados e a explicação de suas potencialidades pode trazer luz a docentes interessados em compreender a educação matemática num cenário de tecnologias digitais de informação e comunicação.

Em especial, o ensino da Geometria Espacial pode ser beneficiado com a adoção de aplicativos em dispositivos móveis que exploram a realidade aumentada na visualização espacial. $\mathrm{O}$ uso de dispositivos móveis como tablets e smartphones desloca o protagonismo da manipulação dos objetos de aprendizagem espaciais para as mãos do estudante, pois este passa a utilizar o seu próprio dispositivo (BYOD). 
Os constructos internos de cada estudante passam a ser afetados pela manipulação em tempo real do objeto espacial, não só observado, mas manipulado pelas mãos e cérebros ativos do sujeito aprendente. $\mathrm{O}$ docente antes direcionado e implicado a usar de estratégias puramente expositivas, no controle de mídias unidirecionais de apresentação, como projetores multimídia e retroprojetores, integra ao seu repertório didático-pedagógico de uma ferramenta cognitiva (JONASSEN, 2007). O que torna possível a construção de contextos de aprendizagem em maior sintonia com a realidade tecnológica atual de nossos estudantes.

Além disso, permitir que os alunos experimentem essas tecnologias na educação possibilita que eles desenvolvam habilidades tecnológicas que são cada vez mais exigidas no contexto profissional, sendo assim mediar o processo entre a educação e o uso da tecnologia é uma forma de preparar os estudantes para os desafios futuros.

\section{REFERÊNCIAS}

AUSUBEL, David. Aquisição e Retenção de Conhecimentos: Uma Perspectiva Cognitiva. Lisboa: Paralelo, 2003.

AZUMA, Ronald. A Survey of Augmented Reality. Teleoperators and Virtual Environments. Los Angeles, n. 6, p. 355 - 385, ago. 1997. Disponível em: <http://ronaldazuma.com/papers/ARpresence.pdf $>$. Acesso em: 08 maio 2018.

BECKER, Fernando; FRANCO, Sérgio Roberto (Org.). Revisitando Piaget. Porto Alegre: Mediação. 1998.

BRASIL. Secretaria de Educação Fundamental. Parâmetros Curriculares Nacionais: ensino de primeira à quarta série. Brasília: MEC/SEF, 1998.

DE ALMEIDA, Caio Augusto Rabite; DE ARAÚJO LIMA, Fernando Tadeu; BORGES, Marcos Martins. Tectônicas Digitais: a (in) tangibilidade no processo de projeto em arquitetura.

Design e Tecnologia, v. 9, n. 18, p. 01-21, 2019.

ESTADO DE MINAS. Brasil tem 230 mi de smartphones em uso. Disponível em: $<$ https:// www.em.com.br/app/noticia/economia/2019/04/26/internas_economia,1049125/brasil-tem-230mi-de-smartphones-em-uso.shtml>. Acesso em: 30 mai. 2020.

EVES, Howard. Introdução à história da matemática. Trad. Hygino H. Domingues. $3^{\mathrm{a}}$ ed. Campinas-SP: Editora UNICAMP, 2004.

\section{plurais}


JONASSEN, David. Computadores, ferramentas cognitivas: desenvolver o pensamento crítico nas escolas. Porto: Porto Editora, 2007.

MARQUES, Vanessa; CALDEIRA, Cláudia Rosana. Dificuldades e carências na aprendizagem da Matemática do Ensino Fundamental e suas implicações no conhecimento da Geometria. Revista Thema, v. 15, n. 2, p. 403-413, 2018.

KIRNER, Cláudio.; KIRNER, Tereza. Evolução e Tendências da Realidade Virtual e da Realidade Aumentada. In: Ribeiro, Marcos Wagner; Zorzal, Ezequiel Roberto (Org.). Realidade Virtual e Aumentada: Aplicações e Tendências. Realidade Virtual e Aumentada: Aplicações e Tendências. Porto Alegre: SBC, 2011, v. 1, p. 8-23.

KIRNER, Cláudio; SISCOUTO, Robson. Fundamentos da Realidade Aumentada. In: KIRNER, Cláudio; SISCOUTO, Robson (Org.). Realidade Virtual e Aumentada: Conceitos, Projeto e Aplicações. Petrópolis: SBC, 2007, p. 2-21. Disponível em: <http://www.redalyc.org/ pdf/5044/504450759004.pdf>. Acesso em: 05 maio 2018.

PIAGET, Jean; GARCIA, Rolando. Psicogênese e História das Ciências. Petrópolis, RJ: Vozes, 2011.

PIAGET, Jean; INHELDER, Bärbel; A representação do espaço na criança. $1^{\circ}$. ed. Porto Alegre: Artes Médicas, 1981.

Recebido em: 19 de junho de 2020.

Inserido em: 10 de agosto de 2020.

Esta obra está licenciada com uma Licença Creative Commons Atribuição 4.0 Internacional. 